\title{
ON THE DETERMINATION OF THE DISTANCE BETWEEN TWO
}

\section{POINTS IN SPACE OF $n$ DIMENSIONS*}

\author{
BY
}

\section{H. F. BLICHFELDT}

\section{Introduction.}

The investigations on the foundation of geometry of Riemans, Hecmeoltz and LIE have as a basal notion the distance between two points of space, and the analytic representation of this distance plays an important rôle. A point in space is determined by means of three independent coördinates; and the distance between two points is a certain function of their six coördinates. Rigid bodies are used and the continuous motions of such, determined by the continuous variation of the coördinates of their points. This notion of three independent and continuous coördinates of a point is, in reality, not simple, as has been pointed out by LIE and others. In an article entitled Ein Beitrag zur Mannigfaltigkeitslehre, Borchhardt's Journal, vol. 84 (1877), G. CANTor describes a space in which the coördinates of a point are neither independent nor continuous.

It does not seem easy, however, to determine the distance between two points of space if the coördinates of a point are neither independent nor continuous. It would not be evident, a priori, that certain loci (as spheres and circles) exist, defined by means of certain equations in the coördinates of their points. Such loci are used in the processes employed by Hesmholtz and Lie, and the question arises whether their axioms involving such loci and the motion of points in space, etc., should be modified or replaced by others.

One such new axiom, used in the following paper, refers to distance-relations, i. e., it is assumed that certain definite relations connect the mutual distances between any $n+2$ different points in space of $n$ dimensions. $\dagger$

One notices that the assumption, used by HeLmholtz and LIE and their successors, that the distance between two points in space is a certain function of the

\footnotetext{
* Presented to the Society (San Francisco) May 3, 1902. Received for publication July 24, 1902.

† Such an axiom has been given by M. J. DE TILLY in his Essai de géométrie analytique générale, Mémoirescouronnés et autres mémoires publiés par l'Académie Royale etc. de Belgique, vol. 47 (1892-93).
} 
six coördinates of the two points, implies the existence of distance-relations ; for between the mutual distances (28) of eight points we could obviously eliminate the 24 coördinates involved, giving us at least four general relations.

The following paper is an attempt to define the analytic form of the distance between two points by means of a set of axioms, based on the assumption that distance-relations exist. The continuity and independence of the coördinates used will not be assumed. A system of typical differential equations will be found - the same found by LiE - which are satisfied by the functions representing the distance.

Some remarks regarding the definitions given below may be in place here. It is not necessary for us to suppose that space is a continuum of points, as this phrase is generally understood. We may, for instance, select all the points in ordinary euclidean space whose cartesian coördinates are integers only. The distances need not form a continuous series of numbers.

In order to deal with known functions we shall at the outset confine ourselves to algebraic distance-relations, though a much wider range of relations may be treated in practically the same manner.

The analysis given does not discriminate between actual distance and any function of this distance. We shall, therefore, understand that the distance-relations are algebraic in the actual distances (as in euclidean space) or in certain transcendental functions of the distances (as in hyperbolic or elliptic space). If the latter is the case, such transcendental functions of the distances will be termed the distances themselves; and in the former case it is sometimes convenient to use the word distance for a certain algebraic function of the actual distance.

It must be borne in mind that the purpose of this paper is the discovery of the functions that may represent the distance between two points in a space defined by means of certain axioms given below, and not a discussion of the geometric properties of such a space. Certain properties of the functions referred to are found in $\S 2$, and here nothing is assumed in regard to continuity of the coördinates involved. The distances may enjoy certain additional properties if the coördinates be supposed continuous and independent, and such properties are found (Theorems 5 and 6 ) which, in connection with the Theorems 1-4 form the bases for the succeeding paragraphs, in which the coördinates are throughout considered continuous and independent. Infinitesimal transformations are here introduced for the purpose of analysis, and have no reference to geometric transformations of points of the spaces considered.

\section{$\S 1$. Definitions and Axioms.}

Art. 1. Definitions.

1. Space (of any number of dimensions) is a collection of an unlimited number of points. 
2. To any pair of points of space corresponds a real number, in general finite, called the distance between the two points. If these be designated $a$ and $b$, we shall denote the distance between them by $\overline{a, b}$, or $\overline{b, a}$.

3. For a certain integer $m$ the mutual distances between any $m$ points of a given space may, without exceptions, satisfy certain definite, algebraic relations. Such relations are then called m-point distance-relations, or simply distancerelations.

4. Space of $n$ dimensions is a space for which $(n+2)$-point distance-relations (rational and integral) exist, but no such $k$-point distance-relations, if $k<n+2$. The symbol $S_{n}$ means "space of $n$ dimensions."

Art. 2. Axioms.

I. For any $n>0$, a space of $n$ dimensions exists.

II. If a finite number of points be cut out of a $S_{n}$ the remaining points will still form a $S_{n}$.

III. A $S_{n}$ contains always a $S_{n-1}$, if $n>1$.

IV.* We shall assume that such $m$ points (fixed) $1,2, \cdots, m,(m \leqq n)$, of any given $S_{n}$ - denoted by $(A)$ - may be selected, and such a $S_{n}$, contained in $(A)$, and denoted by $(B)$, may be defined, that,

$1^{\circ}$. The distance $\overline{a, b}-a, b$ being any pair of points of $(B)$ - can be expressed as a uniform, algebraic function of the $2 m$ distances $\overline{1}, a, \overline{2}, a, \ldots, \overline{m, a}$; $\overline{1, b}, \overline{2, b}, \ldots, \overline{m, b}$, supposed algebraically independent of each other. (The functions considered can, for example, be expanded in a convergent power-series in the distances $1, a, \ldots, \overline{m, b}$, as long as $a, b$ remain within $(B))$;

$2^{\circ}$. For any $S_{n}$ contained in $(B)$, there is no algebraic equation satisfied by the distances $\overline{1, a}, \overline{2}, a, \ldots, \overline{m, a}, a$ being any point of this $S_{n}$.

$3^{\circ}$. Given an algebraic equation in the distances $\overline{1}, \overline{2}, \overline{1,}, \overline{3}, 2, \overline{3}, \ldots, \overline{n-1, n}$; the points $1,2,3, \ldots, n$ may be selected such that this equation is not satisfied.

Definition 5 . The distances $\overline{1, t}, \overline{2, t}, \ldots, \overline{m, t}$ between any point $t$ of $(B)$ and the points $1,2, \ldots, m$ shall be called the coordinates of the point $t$, and shall be denoted by $x_{1}^{t}, x_{2}^{t}, \cdots, x_{m}^{t}$, or simply by $x_{1}, x_{2}, \cdots, x_{m}$.

Thus, to every point $t$ of $(B)$ we have a set of $m$ coördinates. It is not necessarily true, however, that a set of values arbitrarily assigned to the numbers $x_{1}^{t}, x_{2}^{t}, \ldots, x_{m}^{t}$ will make them coördinates of a point of $(B)$, nor is it necessarily true that two points of $(B)$ may be found whose coördinates differ by infinitesimals.

* By means of the alyebraic $(n+2)$-point distance-relations, one, at least, of the $\frac{1}{2}(n+2)(n+1)$ distances between any $n+2$ points of a given ' $n$ is an algebraic function of the remaining distances. The nature of this function, when some of the $n+2$ points occupy definite positions, is more particularly defined by means of this Axiom (IV). 
By Axiom IV, for points $a, b$ of $(B)$ the distance $\bar{a}, b$ is a uniform algebraic function of the $2 m$ coördinates of the points $a$ and $b$, say

$$
\overline{a, b}=\phi\left(x_{1}^{a}, x_{2}^{a}, \cdots, x_{m}^{a} ; x_{1}^{b}, x_{2}^{h}, \cdots, x_{m}^{b}\right) .
$$

\section{§ 2. ThEOREMS.}

Art 3. Let $a, b, c, \cdots$ be any $n+2$ different points of $(B)$, and let

$$
\psi(\overline{a, b}, \overline{a, c}, b, c, \cdots)=0
$$

be one of the $(n+2)$-point distance-relations holding throughout $(A)$; the function $\psi$ is a rational integral function of its $\frac{1}{2}(n+2)(n+1)$ arguments.

1. The $\frac{1}{2}(n+2)(n+1)$ distances $a, b, a, c, \bar{b}, c, \cdots$, written in the coördinates of the points $a, b, c, \cdots$, must satisfy identically the equation $\psi=0$.

Suppose, after substitution in $\psi=0$ of the distances $a, b$, etc., in terms of the coördinates $x_{1}^{n}, x_{1}^{b}$, etc., there results an equation $\rho=0$ connecting the $m(n+2)$ coördinates considered.

By taking for the point $a$ of $(B)$ a definite point, so that the coördinates $x_{1}^{n}, x_{2}^{n}, \ldots, x_{m}^{n}$ are definite constants, this algebraic. equation becomes an identity or an equation in the $m(n+1)$ coördinates of any $n+1$ points $b, c, \cdots$ of the $S_{n}^{\prime}$ obtained by cutting out of $(B)$ the point $a$. In case it becomes an identity if the $n(n+1)$ coördinates $x_{1}^{b}, x_{2}^{\prime}, \cdots, x_{m}^{\prime \prime}, x_{1}^{r}, \cdots$ be considered arbitrary variables, and the point $a$ selected be any point whatever of $(B)$, we can give to the coördinates $x_{1}^{b}, x_{2}^{b}, \ldots, x_{m}^{h} ; x_{1}^{r}, \ldots$ in $\rho=0$ arbitrary constant values, and will thereby obtain an equation in the coördinates $x_{1}^{n}, x_{2}^{n}, \ldots, x_{n}^{n}$ true for every point $a$ of $(B)$. Thus, from an equation $\rho=0$ connecting the coördinates of $n+2$ points of $(B)$ will result an equation connecting the coördinates of less than $n+2$ points of $(B)$, or of a $S_{n}$ contained in $(I)$. The process of reduction may be carried on till we have obtained an algebraic equation $\sigma=0$ in the $m$ coördinates of any point of a $S_{n}$ contained in $(I)$. But, by Axiom IV, no such equation can exist. The theorem is therefore true.

2. The number $m$ used in Axiom IV must be equal to $n$; $i$. e., the $n$ distances between the points $1,2, \cdots, n$ of $(A)$ and any point of $(B)$ are algebraically independent of each other.

According to Axiom III, the space $(B)$ contains a $S_{n-1}$, which we shall call $(C)$. Let $\chi(a, b, a, c, b, c, \cdots)=0$ be one of the algebraic relations connecting the mutual distances of $n+1$ points $a, b, c, \cdots$ of $(c)$. This equation is not identically satisfied if we substitute for $\bar{a}, b, \bar{a}, c, \ldots$ their values in terms of the $m(n+1)$ coördinates $x_{1}^{n}, \ldots$, or the equation $\chi=0$ would hold throughout $(B)$. Hence, an algebraic equation $\rho^{\prime}=0$ results, from which, as from the 
equation $\rho=0$ above, we find an algebraic equation connecting the coördinates of any point of $(C)$ or of a $S_{n-1}$ contained in $(C)$.

According to Axiom IV, we can select a new system of coördinates for this $S_{n-1}$, say $y_{1}, y_{2}, \cdots, y_{k}$, which represent distances between $k$ definite points of $(C)$ and any point of a $S_{n-1}(D)$ contained in $(C)$. All these points lying in $(C)$, their distances are functions of the former system of coördinates, and the coördinates $y_{1}^{t}, y_{2}^{t}, \cdots, y_{k}^{t}$ of any point $t$ of $(D)$ are given in terms of definite constants and of the coördinates $x_{1}^{t}, x_{2}^{t}, \ldots, x_{m}^{t}$. At most $m-1$ of these are algebraically independent for points of $(C)$, as we have seen, and therefore $k \leqq m-1$, since the numbers $y_{1}^{t}, y_{2}^{t}, \cdots, y_{k}^{t}$ are algebraically independent.

The space $(D)$ contains a $S_{n-2}$, say $(E)$. For this space we have a system of coördinates $z_{1}, z_{2}, \cdots, z_{l}$. We find $l \leqq k-1$.

Proceeding thus, we get a series of spaces of dimensions

$$
n, n-1, n-2, \cdots, 1
$$

with systems of coördinates of numbers

with the relations

$$
m, k, l, \ldots, v \text {, }
$$

$$
n \geqq m>k>l>\cdots>v>0 \text {. }
$$

The number $v$ (for space of one dimension) is 1 at least, as all distances in that space would otherwise be the same constant. This is impossible for a space of one dimension, by Definition 4 . It follows that

$$
v=1, \cdots, l=n-2, k=n-1, m=n .
$$

3. In any $S_{n}$, but one $(n+2)$-point algebraic distance-relation exists.

For, if we had more than one such, we would have two or more relations connecting the distances between the points $1,2, \ldots, n, a, b$ used in Axiom IV, from which would follow one or more algebraic relations connecting the distances $\overline{1,2}, \overline{1,3}, \ldots, \overline{n-1, n}$ and the $2 n$ coördinates of the two points $a, b$, which could not be free from the latter by Axiom IV, part $3^{\circ}$, the points $1,2,3, \cdots, n-1, n$ being properly chosen. Such relations would, as we have seen, lead to relations connecting the $n$ coördinates of any one point of a $S_{n}$. Hence the theorem.

4. The function

$$
\overline{a, b}=\phi\left(x_{1}^{a}, x_{2}^{\prime \prime}, \ldots, x_{n}^{\prime \prime} ; x_{1}^{b}, x_{2}^{b}, \cdots, x_{n}^{b}\right)
$$

is unaltered if all the coördinates of each point be interchanged with the corresponding coirrdinates of the other. 
The contrary hypothesis would, namely, lead to an algebraic equation connecting the coördinates of any one point, following from

$$
\overline{a, b}=\overline{b, a}=\phi\left(x_{1}^{a}, \cdots, x_{n}^{a} ; x_{1}^{b}, \cdots, x_{n}^{b}\right)=\phi\left(x_{1}^{b}, \cdots, x_{n}^{b} ; x_{1}^{a}, \cdots, x_{n}^{a}\right) .
$$

5. If $1^{\prime}, 2^{\prime}, 3^{\prime}, \cdots, n^{\prime}, a$ are points of $(B)$, then are the distances

$$
\overline{1^{\prime}, a}, \overline{2^{\prime}, a}, \cdots, \overline{n^{\prime}, a}
$$

independent of each other with respect to the coördinates $x_{1}^{n}, x_{2}^{n}, \ldots, x_{n}^{a}$; these, as well as the coördinates $x_{1}^{1^{\prime}}, \ldots, x_{n}^{1^{\prime}} ; \ldots ; x_{1}^{n^{\prime}}, \ldots, x_{n}^{n^{\prime}}$ being considered inclependent variables.

We may, according to Axiom IV, choose new coördinates $y_{1}, y_{2}, \ldots, y_{n}$ for a $S_{n}$, which we shall call $(G)$, contained in $(B)$. These coördinates represent the distances from $n$ definite points, say $1^{\prime}, 2^{\prime}, \cdots, n^{\prime}$, of $(B)$ and any given point of $(G)$. The points $1^{\prime}, 2^{\prime}, \cdots, n^{\prime}$ may, moreover, be chosen so that the distances

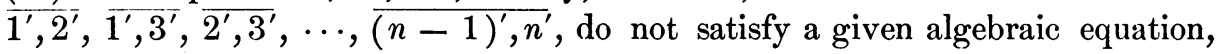
$\sigma=0$. This equation we shall determine in the following manner :

Let, for the moment, $1^{\prime}, 2^{\prime}, \ldots, n^{\prime}$ be arbitrary points of $(B)$, and let $a, b, c, \cdots$ be other $\frac{1}{2} n(n+1)+1$ points of $(B)$. Suppose Theorem 5 not true; then we would have one or more algebraical identities of the form

$\zeta\left(\overline{1^{\prime}, a}, \overline{2^{\prime}, a}, \ldots, \overline{n^{\prime}, a} ; x_{1}^{1^{\prime}}, x_{2}^{1^{\prime}}, \cdots, x_{n}^{1^{\prime}} ; x_{1}^{2^{\prime}}, \ldots, x_{n}^{2^{\prime}} ; \ldots ; x_{1}^{n^{\prime}}, x_{2}^{n^{\prime}}, \ldots, x_{n}^{n^{\prime}}\right)=0$,

and similar equations for the points $b, c, \ldots$. Between these and the $\frac{1}{2} n(n-1)$ equations

$\overline{1^{\prime}, 2^{\prime}}=\phi\left(x_{1}^{1^{\prime}}, x_{2}^{1^{\prime}}, \cdots, x_{n}^{1^{\prime}} ; x_{1}^{2^{\prime}}, x_{2}^{2^{\prime}}, \cdots, x_{n}^{2^{\prime}}\right), 1^{\prime}, 3^{\prime}=$ etc., $\cdots,(n-1)^{\prime}, n^{\prime}=$ etc.,

we can eliminate the $n^{2}$ coördinates $x_{1}^{1^{\prime}}, \ldots, x_{n}^{n^{\prime}}$ involved, thereby obtaining at least one algebraic equation $\tau=0$ connecting the distances

$$
\overline{1^{\prime}, \overline{2^{\prime}}}, \overline{1^{\prime}, 3^{\prime}}, \cdots, \overline{(n-1)^{\prime}, n^{\prime}} ; \overline{1^{\prime}, a}, \ldots, \overline{n^{\prime}, a} ; \overline{1^{\prime}, b}, \ldots, n^{\prime}, b \text {, etc. }
$$

Let us, in this equation, substitute arbitrary, but definite constants for the distances $\overline{1^{\prime}, a}, \overline{2^{\prime}, a}, \cdots, \overline{n^{\prime}, a} ; \overline{1^{\prime}, b}, \overline{2^{\prime}, b}$, etc. We get an equation in the distances $\overline{1^{\prime}, 2^{\prime}}, \overline{1^{\prime}, 3^{\prime}}, \overline{2^{\prime}, 3^{\prime}}, \ldots, \overline{(n-1)^{\prime}, n^{\prime}}$, which is not satisfied in general, as the points $1^{\prime}, 2^{\prime}, \ldots, n^{\prime}$ are taken from a $s_{n}^{\prime}$ (Definition 4 ), but might be satisfied for special points of $(B)$. This is the equation we shall call $\sigma=0$, and the points $1^{\prime}, 2^{\prime}, \ldots, n^{\prime}$ shall be chosen so that it is not satisfied. If so, the equation $\tau=0$ cannot be an identity, but is an actual equation in the distances $\overline{1^{\prime}, a}, 2^{\prime}, a, \cdots, \overline{n^{\prime}, a} ; \overline{1^{\prime}, b}$, etc., which are now the coördinates $y_{1}^{n}, \cdots, y_{n}^{\prime \prime}$, etc., of the points $a, b, c, \cdots$, of $(G)$. But, the existence of an algebraic equation connecting the coördinates of any given number of arbitrary points of $(G)$ leads to an algebraic equation connecting the coördinates of any point of a $S_{n}$ contained in $(G)$. Such an equation cannot exist by Theorem 2. Hence the Theorem 5 . 
6. The distance $\overline{a, b}=\phi\left(x_{1}^{a}, \ldots, x_{n}^{a} ; x_{1}^{b}, \ldots, x_{n}^{b}\right)$ does not satisfy a linear partial differential equation of the form

$$
X(\overline{a, b}) \equiv A_{1} \frac{\partial(\overline{a, b})}{\partial x_{1}^{a}}+A_{2} \frac{\partial(\overline{a, b})}{\partial x_{2}^{a}}+\cdots+A_{n} \frac{\partial(\overline{a, b})}{\partial x_{n}^{a}}=0,
$$

where the coefficients $A_{1}, A_{2}, \cdots, A_{n}$ are functions of $x_{1}^{n}, x_{2}^{n}, \cdots, x_{n}^{a}$ only.

Assume the existence of such an equation, and suppose $A_{n} \neq 0$. Let $1,2, \cdots, n, a$ be auy $n+1$ points of $(B){ }^{*}$ Between the equations

$$
\left\{\begin{array}{c}
\overline{1, a}=\phi\left(x_{1}^{1}, \cdots, x_{n}^{1} ; x_{1}^{a}, \cdots, x_{n}^{a}\right), \\
\overline{2, a}=\phi\left(x_{1}^{2}, \cdots, x_{n}^{2} ; x_{1}^{a}, \ldots, x_{n}^{a}\right), \\
\left.\cdot \cdot \cdot \cdot \cdot \cdot \cdot \cdot \cdot x_{n}^{a}\right)
\end{array}\right.
$$

can be eliminated just $n-1$ of the coördinates $x_{1}^{n}, x_{2}^{a}, \ldots, x_{n}^{a}$, say $x_{1}^{n}, \cdots, x_{n-1}^{a}$ (Theorem 5). The resulting equation,

$$
\xi\left(\overline{1, a}, \overline{2, a}, \cdots, \overline{n, a} ; x_{n}^{a} ; x_{1}^{1}, \cdots, x_{n}^{1} ; \cdots ; x_{1}^{n}, \cdots, x_{n}^{n}\right)=0,
$$

algebraic in

$$
\overline{1, a}, \overline{2, a}, \cdots, \overline{n,}, \bar{a}, x_{n}^{a}, x_{1}^{\mathrm{1}}, \cdots, x_{n}^{n}
$$

becomes an identity upon the substitution of the values of $1, a, 2, a, \cdots, \overline{n, a}$ from (3). Differentiating this identity in turn with respect to $x_{1}^{n}, x_{2}^{a}, \cdots, x_{n}^{a}$ and multiplying successively by $A_{1}, A_{2}, \cdots, A_{n}$, we obtain

since

$$
X(\xi) \equiv A_{n} \frac{\partial \xi}{\partial x_{n}^{a}} \equiv 0
$$

$$
X(1, a) \equiv X(2, a) \equiv \cdots \equiv X(n, a) \equiv 0
$$

Supposing $\xi$ to be integral and irreducible, we can not have $\partial \xi / \partial x_{n}^{n} \equiv 0$. Accordingly, $A_{n}=0$, contrary to hypothesis.

\section{§3. Construction of the Differential Equations.}

Art. 4. Let us take $n+2$ arbitrary points of $(B)$ and designate them

$$
1,2, \cdots, n ; a, b \text {. }
$$

Just one algebraic relation connects the $\frac{1}{2}(n+1)(n+2)$ mutual distances between these points, and this relation is satisfied identically upon the substitu-

* The coördinates $x_{1}^{a}, x_{2}^{a}, \cdots, x_{n}^{a}$ represent the distances between the point $n$ of $(B)$ and $n$ points of $(A)$ which we denoted by $1,2, \cdots, n$. As these points are not referred to in the future, we shall, for convenience, hereafter reserve the numbers $1,2, \cdots, n$ for points of $(B)$. 
tion of the algebraic functions of the form (1) for the distances (Theorems 1-3). For the purpose of finding these functions we may therefore regard the $n$ coördinates of any point as independent and continuous variables. If we do this, the $\frac{1}{2}(n+1)(n+2)$ functions considered will satisfy only one relation, and as they involve $n(n+2)$ coördinates, we can construct a system of just

$$
n(n+2)-\left[\frac{1}{2}(n+1)(n+2)-1\right]=\frac{1}{2} n(n+1)
$$

independent linear partial differential equations whose solutions are the distances considered. Let this system be indicated by $M$.

For simplicity, we shall write $p_{i}^{a}$ instead of $\partial f / \partial x_{i}^{a}, f$ being any solution of $M$. Each of the equations of this system is therefore linear and homogeneous in the letters $p_{i}^{a}, i=1,2, \cdots, n ; \alpha=1,2, \ldots, n, a, b$, and the coefficients are algebraic functions of the variables $x_{1}^{1}, \ldots, x_{n}^{n} ; x_{1}^{a}, \ldots, x_{n}^{a} ; x_{1}^{b}, \ldots, x_{n}^{b}$.

The functions $\overline{1}, \bar{a}, \ldots, \bar{n}, a$ being independent with respect to $x_{1}^{n}, \ldots, x_{n}^{a}$ (Theorem 5), we may make a change of variables in $M$, replacing the variables

$$
x_{1}^{n}, x_{2}^{\prime \prime}, \ldots, x_{n}^{a} ; x_{1}^{b}, x_{2}^{b}, \ldots, x_{n}^{b},
$$

involved in $M$, by the variables

$$
1, \bar{a}, \overline{2, a}, \ldots, \overline{n, a} ; \overline{1, b}, \overline{2, b}, \ldots, \overline{n, b} .
$$

The resulting system $\left(M^{\prime}\right)$ of differential equations will differ from $M$ merely in being free from the derivatives $p_{i}^{a}, p_{i}^{b}, i=1,2, \ldots, n$, and in having the variables in the coefficients changed. As the system $M$ contains $\frac{1}{2} n(n+1)$ independent equations, the new system $M^{\prime}$ must contain $\frac{1}{2} n(n+1)$ independent equations, which involve the derivatives $p_{i}^{a} ; i, \alpha=1,2, \cdots, n$, only. Accordingly, we can solve $M^{\prime}$ for $\frac{1}{2} n(n+1)$ of these derivatives, and therefore also $M$.

Art. 5. Solving the system $M$ in this manner, let the general solution be indicated by

$$
p_{j}^{\beta}=[n]+(a)+(b),
$$

the derivatives $p_{j}^{\beta}$ being $\frac{1}{2} n(n+1)$ of the derivatives $p_{i}^{\alpha} ; i, \alpha=1,2, \ldots, n$, the remaining of which are included in the symbol $[n]$. The symbols $(a)$ and (b) represent respectively the terms containing the derivatives $p_{i}^{n}$ and $p_{i}^{h}, i=1,2, \cdots, n$, and are derived one from the other by interchanging all the corresponding variables and derivatives of the two points $a$ and $b$. For, the distances $\overline{\alpha, \beta} ; \alpha, \beta=1,2, \ldots, n, a, b ; \alpha \neq \beta$, defining the system (4), are unaltered or interchanged by interchanging all the corresponding coördinates of any two of the points $1,2, \ldots, n, a, b$ (Theorem 4). It follows from this that the system of equations (4) are reproduced identically by interchanging the corresponding coördinates of the two points $a$ and $b$. 
The $\frac{1}{2} n(n+1)$ equations indicated by

$$
p_{j}^{\beta}=[n]+(a)
$$

are independent and are satisfied by the $\frac{1}{2} n(n+1)$ independent distances $\overline{\alpha, \beta} ; \alpha, \beta=1,2, \cdots, n, \alpha ; \alpha \neq \beta$, which are free from the coördinates $x_{1}^{b}, x_{2}^{b} \cdots, x_{n}^{b}$. As only $\frac{1}{2} n(n+1)$ independent linear partial differential equations can be constructed having for solutions $\frac{1}{2} n(n+1)$ independent functions in $n(n+1)$ variables

$$
x_{1}^{1}, \ldots, x_{n}^{1} ; x_{1}^{2}, \ldots, x_{n}^{2} ; \cdots ; x_{1}^{n}, \cdots, x_{n}^{n} ; x_{1}^{a}, \cdots x_{n}^{a},
$$

it is easily proved that the equations (5) must be free from the variables $x_{1}^{b}, x_{2}^{b}, \ldots, x_{n}^{b}$; i. e., the terms indicated by the symbol $(a)$ do not contain the coördinates $x_{1}^{b}, \cdots, x_{n}^{b}$. Similarly, the terms indicated by the symbol $(b)$ do not contain the coördinates $x_{1}^{a}, \cdots, x_{n}^{a}$.

The system of equations

$$
(a)+(b)=0
$$

must evidently be satisfied by the distance $\overline{a, b}$, whatever be the values of the coördinates $x_{i}^{a} ; i, \alpha=1,2, \cdots, n$, which do not occur in $\overline{a, b}$, but are, likely, involved in the coefficients of $(a)$ and $(b)$. Giving all possible sets of constant values to these $n^{2}$ coördinates, we derive from (6) several such equations, $l$ in number, suppose, not satisfying linear relations with constant coefficients. Let any one of these $l$ equations be indicated by

$$
[(a)]+[(b)]=0 .
$$

Art. 6. If we now form the system of $l$ equations

$$
X_{i}(f) \equiv[(1)]+[(2)]+\cdots+[(n)]+[(a)]+[(b)]=0 \quad(i=1,2, \cdots, l),
$$

we readily see that it is satisfied by all the $\frac{1}{2}(n+1)(n+2)$ distances $\overline{\alpha, \beta} ; \alpha, \beta=1,2, \cdots, n, a, b ; \alpha \neq \beta$. Let $k$ of them be independent. They may then replace $k$ of the system (4), and the remaining $\frac{1}{2} n(n+1)-k$ equations can be taken so as to be free from the letters

$$
p_{i}^{a}, p_{i}^{b}, x_{i}^{a}, x_{i}^{b} \quad(i=1,2, \cdots, n),
$$

owing to the form of the equations (4). Indeed, the coefficients involved in (a) are readily seen to be of the form $\sum_{i} A_{i}^{\prime} F_{i}$, where $A_{i}^{\prime}$ is a function of $x_{1}^{a}, x_{2}^{a}, \cdots, x_{n}^{a}$ only, and $F_{i}$ is free from these letters and from $x_{1}^{b}, x_{2}^{b}, \cdots, x_{n}^{b}$; the coefficients in $(b)$ have the same form if $a$ and $b$ be interchanged.

These remaining equations must be satisfied by the distances

$$
\overline{a, a}
$$

$$
(a=1,2, \cdots, n) .
$$


Substituting we find, for some value of $\alpha$, an equation of the form

$$
\sum_{i=1}^{n} A_{i} \frac{\partial(\overline{\alpha, a})}{\partial x_{i}^{a}}=0
$$

where the coefficients $A_{i}$ do not contain the coördinates $x_{1}^{n}, \ldots, x_{n}^{n}$ as may be easily proved by the reader from what precedes. All other coördinates, excepting $x_{1}^{a}, \ldots, x_{n}^{a}$; may be put equal to arbitrary constants. There remains an equation of the form debarred by Theorem 6 . Accordingly, $k=\frac{1}{2} n(n+1)$, and the system (4), or $M$, is replaced completely by the system (7).

Let the first $\frac{1}{2} n(n+1)$ of the equations (7) be independent. They form what is called a complete system of linear partial differential equations, as

$$
n(n+2)=\left[\frac{1}{2} n(n+1)\right]+\left[\frac{1}{2}(n+1)(n+2)-1\right] .
$$

Therefore, by the theory of linear partial differential equations, the " commutator" of every pair of the left-hand members of $(7)$, as $X_{i}(f)$ and $X_{j}(f)$,

$$
X_{i}\left(X_{j}(f)\right)-X_{j}\left(X_{i}(f)\right)
$$

must be linearly dependent upon the expressions

namely,

$$
X_{i}(f) \quad\left[i=1,2, \cdots, \frac{1}{2} n(n+1)\right],
$$

$$
\begin{gathered}
X_{i}\left(X_{j}(f)\right)-X_{j}\left(X_{i}(f)\right) \equiv \sum_{s=1}^{k} \alpha_{s} X_{s}(f) \\
{\left[i, j=1,2, \cdots, k ; i \neq j, k=\frac{1}{2} n(n+1)\right] .}
\end{gathered}
$$

These commutators are immediately seen to have the same form as the expressions $X_{i}(f)$, i. e., they have the form

$$
[(1)]+[(2)]+\cdots+[(n)]+[(a)]+[(b)],
$$

in which the symbol $[(\alpha)]$ represents a linear and homogeneous expression in the derivatives $p_{i}^{a}, i=1,2, \cdots, n$, with coefficients which are functions of $x_{i}^{a}, i=1,2, \cdots, n$, only. Moreover, the expressions represented by two symbols $[(\alpha)]$ and $[(\beta)]$ are obtained one from the other by interchanging all the corresponding coördinates of the points $\alpha$ and $\beta$.

We can now prove that the conditions (8) can be satisfied only if the coefficients $\alpha_{s}$ are constants. For, if not, the integer $v>n$ can be taken great enough that the $\frac{1}{2} n(n+1)+1$ equations of the form

$$
X_{i}(f) \equiv[(1)]+[(2)]+\cdots+[(v)]=0 \quad(i=1,2, \cdots, k),
$$


obtained from the $k$ first of the system (7) and one of the commutators (8) are independent, as the reader may readily prove. The distances

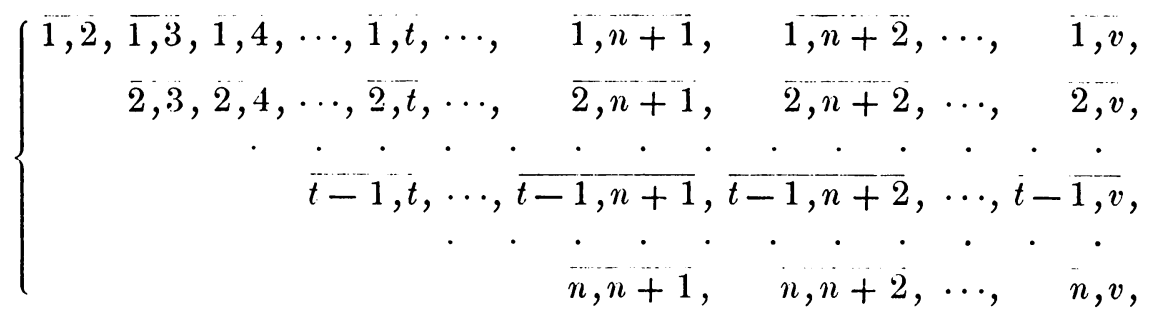

are solutions of this system and are all independent. For, if they were not, the distances $\overline{1}, w, 2, w, \ldots, n, w$ would satisfy a relation free from the variables $x_{1}^{r r}, x_{2}^{r r}, \ldots, x_{n}^{r r}$, contrary to Theorem $5, w$ being the highest number occurring in the distances satisfying a tentative relation. (If $w<n+1$, omit $\bar{w}, \bar{w}$.) The number of solutions (10) being $n v-\frac{1}{2} n(n+1)$, we would have a contradiction, as $\frac{1}{2} n(n+1)+1$ independent equations in $n v$ variables have only $n v-\frac{1}{2} n(n+1)-1$ independent solutions.

Art. 7. Consider now the $\frac{1}{2} n(n+1)$ expressions indicated by the symbol $[(1)]$ of the first $\frac{1}{2} n(n+1)$ equations of $(7)$. Omitting the index 1 from the variables and derivatives, they are of the form

$$
Y(f) \equiv A_{1} p_{1}+A_{2} p_{2}+\cdots+A_{n} p_{n},
$$

the coefficients $A_{i}$ being algebraic functions of $x_{1}, x_{2}, \cdots, x_{n}$, and the letters $p_{i}$ being the partial derivatives of $f$ with respect to the same coördinates. We have found that the commutator of any pair of the expressions (11) is equal to a sum of these expressions multiplied by constants. If we denote the $\frac{1}{2} n(n+1)$ expressions (11) by $Y_{1}(f), Y_{2}(f), \cdots, Y_{k}(f)$, where $k=\frac{1}{2} n(n+1)$, we have

$$
Y_{i}\left(Y_{j}(f)\right)-Y_{j}\left(Y_{i}(f)\right) \equiv \sum_{s=1}^{k} c_{i j s} Y_{s}(f) \quad(i, j=1,2, \cdots, k ; i \neq j),
$$

the coefficients $c_{i j s}$ being constants.

The expressions $Y_{1}(f), \cdots, Y_{k}(f)$ can therefore, according to LIF, be considered independent infinitesimal transformations of a $k$-parametric group, $k=\frac{1}{2} n(n+1)$. This group must possess the following properties :

1. It must possess one, and only one, invariant for two points, which is algebraic by a proper choice of variables; i. e., if $Y^{(a)}(f)$ is any infinitesimal transformation of the group, written in the variables $x_{1}^{\alpha}, x_{2}^{\alpha}, \ldots, x_{n}^{\alpha}$, then must the $k$ equations

$$
Y^{(1)}(f)+Y^{(2)}(f)=0
$$

possess one solution only, which must be algebraic. 
2. The condition of Theorem 6 must be observed; i. e., it must be impossible from the $k$ equations (12) to derive a differential equation written only in the variables and derivatives with the index 1 . In particular, no two independent infinitesimal transformations can differ only by a factor, for from two such we would get an equation of the form excluded by Theorem 6 .

The first condition is readily seen to be true by considering the list of solutions (10) of the equations (9). Only one solution can be obtained from that list containing no other variables than $x_{1}^{1}, x_{2}^{1}, \ldots, x_{n}^{1} ; x_{1}^{2}, x_{2}^{2}, \ldots, x_{n}^{2}$.

\section{$\S 4$. Coincidence of the Groups Defined in Art. 7 with a Class of Groups Defined by Lie.}

Art. 8. In his Untersuchungen über die Grundlagen der Geometrie, * LiE has defined a class of groups in the following manner: Two points have one, and only one, invariant; $s$ points, $s>2$, have no invariants independent of the two-point invariants. Such groups have been determined by LIE for $S_{1}, S_{2}$ and $S_{3}, \dagger$ and by Dr. G. Kow.ALEwski for $S_{4} . t$

We proceed to show that our groups as defined by the conditions 1 and 2 of Art. 7, and the groups defined by LIE are identical, if the algebraic character of the distances (invariants) be disregarded. The groups have the same number of parameters when they are written in the same number of variables $n$, namely $\frac{1}{2} n(n+1)$. Let

$$
X_{i}(f) \equiv[(1)]+[(2)]+\cdots+[(s)] \quad\left[i=1,2, \cdots, \frac{1}{2} n(n+1)\right]
$$

represent the differential equations obtained from our groups or from LIE's groups and corresponding to the $\frac{1}{2} n(n+1)$ independent equations of the system (7), $s$ being taken large enough that they are all independent of each other, and so that the invariants of this system contain at least one of the invariants if such exist - that do not depend on the two-point invariants. We may take $s>n$. Consider the $n s-\frac{1}{2} n(n+1)$ invariants (distances) (10) $(v$ replaced by $s$.

They are obviously independent of each other for the groups defined by LIE, and in such a case are the invariants $\overline{1, s}, \overline{2, s}, \ldots, \overline{n, s}$ independent of each other with regard to the variables $x_{1}^{s}, x_{2}^{s}, \cdots, x_{n}^{s}$. Now, this property leads to our condition 2, Art. 7. For, the existence of a differential equation of the form

$$
A_{1} \frac{\partial f}{\partial x_{1}^{s}}+A_{2} \frac{\partial f}{\partial x_{2}^{s}}+\cdots+A_{n} \frac{\partial f}{\partial x_{n}^{s}}=0
$$

* Theorie der Transformationsgruppen, LIE-ENGEL, vol. III, Abtheilung V.

$\dagger$ Theorie der Transformationsgruppen, vol. III, p. 6 and pp. 399-437.

$\ddagger$ Ueher eine Kategorie von Transformationsgruppen einer vierdimensionalen Mannigfultigkiten, Leipziger Berichte, vol. 50, pp. 60-111. 
(the coefficients $A_{i}$ being functions of $x_{1}^{n}, \cdots, x_{n}^{s}$ only), and satisfied by the invariants $\overline{1, s}, \overline{2, s}, \ldots, \overline{n, s}$ would deny the independence of these. LiE's groups therefore satisfy our condition 2 , and evidently also the condition 1 if we disregard the algebraic form of the invariants.

It remains for us to show that our groups defined by the conditions 1 and 2 possess no invariants (solutions of the system (13)), independent of the twopoint invariants (distances).

Firstly, the distances (10) ( $v$ replaced by $s$ ) are independent of each other, if our condition 2 is satisfied. If they were not, the distances $\overline{1, s}, \overline{2, s} \ldots, \overline{n, s}$ would satisfy a relation free from the variables $x_{1}^{s}, x_{2}^{s}, \ldots, x_{n}^{s}$. Now, the distances $\overline{1, s}, \overline{2, s}, \ldots, \overline{n-1, s}$ define a differential equation of the form (14) with this exception, that we know only that the coefficients $A_{i}$ do not now contain the variables $x_{1}^{n}, x_{2}^{n}, \cdots, x_{n}^{n}$. This equation would also be satisfied by the distance $\overline{n, s}$ in the case supposed. It is, however, with respect to this function, an equation of the form debarred by Theorem 6 . Such an equation could therefore not possibly be derived from our groups, which furnish all the differential equations satisfied by the distance $\overline{n, s}$. Hence, the distances (10) are independent of each other. (It has been assumed that if the functions (10) are not independent, the relation existing among them contains actually one, at least, of the functions $\overline{1, s}, 2, s, \cdots, \overline{n, s}$. This need not be so, but the analysis is the same if for $s$ we substitute $t$, this being the highest number occurring in the distances of (10) satisfying a tentative relation. If $t<n+1$, the symbol $t, t$ must, of course, be omitted.)

Secondly, the distances (10) ( $v$ replaced by $s$ ), being all independent, must form a complete set of solutions to the system (13), as the number of independent solutions can be only $n s-\frac{1}{2} n(n+1)$. This system can therefore not contain a solution independent of the distances, and LIE's definition is satisfied by our groups.

Art. 9. We may now take the two-point invariants of the groups obtained by Lie and Kowalewski for $S_{1}, S_{2}, S_{3}, S_{4}$, selecting those that can be put into algebraic form by a proper choice of variables. Calling the coördinates $x ; x, y$; $x, y, z ; x, y, z, u *$ for spaces of $1,2,3,4$, dimensions respectively, the distance $\overline{1,2}$ must be a function of one or other of the following types:

Space of one dimension.

$$
x_{1}-x_{2} ; \quad x_{2} / x_{1}
$$

* These letters may not represent coördinates in the sense in which such have been defined, i. e., distances between certain fixed points and the variable point, but are certain functions of these distances. To put the groups considered into the typical forms given by LIK and KoWALEWSKI may necessitate a change of variables. 
Space of two dimensions.

Space of three dimensions.

$$
\begin{gathered}
\left(y_{1}-y_{2}\right)\left(x_{1}-x_{2}\right)^{c}, c \neq 0 ; \quad x_{1} y_{2}-x_{2} y_{1} \\
\frac{\left(x_{1}-x_{2}\right)^{2}+\left(y_{1}-y_{2}\right)^{2}+\left(x_{1} y_{2}-x_{2} y_{1}\right)^{2}}{\left(1+x_{1} x_{2}+y_{1} y_{2}\right)^{2}}
\end{gathered}
$$

$$
\begin{gathered}
\left(x_{1}-x_{2}\right)^{2}+\left(y_{1}-y_{2}\right)^{2}+\left(z_{1}-z_{2}\right)^{2} ; \\
\frac{\left(x_{1}-x_{2}\right)^{2}+\left(y_{1}-y_{2}\right)^{2}+\left(z_{1}-z_{2}\right)^{2}+\left(x_{1} y_{2}-x_{2} y_{1}\right)^{2}+\left(x_{1} z_{2}-x_{2} z_{1}\right)^{2}+\left(y_{1} z_{2}-y_{2} z_{1}\right)^{2}}{\left(1+x_{1} x_{2}+y_{1} y_{2}+z_{1} z_{2}\right)^{2}} ; \\
z_{1}+z_{2}-2 \frac{y_{1}-y_{2}}{x_{1}-x_{2}} ; \quad z_{1}-z_{2}+x_{2} y_{1}-x_{1} y_{2} ; \\
z_{1}-z_{2}+\frac{\left(y_{2}-y_{1}\right)^{2}}{2\left(x_{2}-x_{1}\right)} ; \quad z_{1} z_{2}\left(x_{1}-x_{2}\right)\left(y_{1}-y_{2}\right)^{c} ; \quad c \neq 0 .
\end{gathered}
$$

Space of four dimensions.

Let $\gamma=u_{1} u_{2}$,

$$
\begin{aligned}
& \alpha=\left(x_{1}-x_{2}\right)^{2}+\left(y_{1}-y_{2}\right)^{2}+\left(z_{1}-z_{2}\right)^{2}+\left(u_{1}-u_{2}\right)^{2}, \\
& \beta=\left(x_{1} y_{2}-x_{2} y_{1}\right)^{2}+\left(x_{1} z_{2}-x_{2} z_{1}\right)^{2}+\left(x_{1} u_{2}-x_{2} u_{1}\right)^{2}+\left(y_{1} z_{2}-y_{2} z_{1}\right)^{2} \\
& +\left(y_{1} u_{2}-y_{2} u_{1}\right)^{2}+\left(z_{1} u_{2}-z_{2} u_{1}\right)^{2} \text {. }
\end{aligned}
$$

The different types are then

$$
\begin{gathered}
\alpha ; \quad \frac{\alpha+\beta}{\left(1+x_{1} x_{2}+y_{1} y_{2}+z_{1} z_{2}+u_{1} u_{2}\right)^{2}} ; \quad \gamma\left[\alpha-\left(u_{1}-u_{2}\right)^{2}\right] ; \\
\gamma\left(z_{1}-z_{2}+x_{2} y_{1}-x_{1} y_{2}\right) .
\end{gathered}
$$

If the coördinates be considered real numbers, we should add to this list all real functions obtained from those given by an imaginary change of variables. In this manner we would, for instance, obtain the function

$$
\left(x_{1}-x_{2}\right)^{2}+\left(y_{1}-y_{2}\right)^{2},
$$

for space of two dimensions, from the form $\left(y_{1}-y_{2}\right)\left(x_{1}-x_{2}\right)^{c}$.

From each of the types just given we can obtain the corresponding distance-relations by simply eliminating the coördinates involved in the distances concerned. It may be remarked that M. DE TILLY's "condition of six points," stated in his Essai de géométrie analytique générale, mentioned above, will hold for all the distance-relations obtained in this manner for $S_{3}$; in fact, for all those corresponding to the two-point invariants given by LIE in

* This type is given by LIE in the form $z_{1}+z_{2}-\log \left(x_{1}-x_{2}\right)^{2}-c \log \left(y_{1}-y_{2}\right)^{2}$. 
his Theorie der Transformationsgruppen, vol. III, pp. 410. This condition, to which DE TILLY attaches prime importance, is as follows: Let the five-point distance-relation connecting the distances of the points $a, b, c, d, e$ be indicated by $(a b c d e)=0$. This must have such a form that from any three of the relations $(12345)=0,(12346)=0,(12356)=0, \quad(12456)=0, \quad(13456)=0$, $(23456)=0$ must follow the other three. DE Tilly gives only two distancerelations satisfying this condition, namely those existing for the euclidean and non-euclidean spaces, corresponding to the two first distances given above for space of three dimensions.

Art. 10. In conclusion it may be pointed out that in the case of the spaces we have defined and discussed, it is not certain that a given equation among the coördinates will define a locus in the ordinary sense, there may be no points, or a finite number only, whose coördinates satisfy the given equation.

There is, however, always one locus in any $S_{n}$ for which such an equation exists. By Axiom III, a $S_{n-1}$ is contained in the $S_{n}$ considered, and it is clear from Theorem 2 and the arguments following that the $n$ coördinates of the points of this $S_{n-1}$ satisfy an algebraic equation, and only one such. In any $S_{n}$ there is therefore always a $S_{1}$, which we might call a straight line, the $n$ coördinates of whose points satisfy $n-1$ independent algebraical equations.

Stanford UNIVERSTY, July, 1902. 